\title{
Angewandte Landschaftsökologie: Der Weg von der Forschung in die Praxis
}

\section{Thomas Mosimann, Hannover}

\section{Angewandte Landschaftsökologie: immer ein langer Weg}

Der Begriff «Angewandte Landschaftsökologie» ruft die Frage hervor nach dem Unterschied zwischen Landschaftsökologie und Angewandter Landschaftsökologie. Landschaftsökologie ist seit den 60er-Jahren ein wissenschaftliches Arbeitsfeld, das dem heute unvermindert anhaltenden Ruf nach Inter- und Multidisziplinarität entspricht. Bezeichnenderweise liess sich Landschaftsökologie nie einem einzigen Fach zuordnen. Es herrscht heute auch Konsens darüber, dass sie mehr eine Arbeitsperspektive als ein Fach im klassischen Sinne ist, also in fast selbstverständlicher Weise modernen Konzepten fächerübergreifenden Arbeitens entspricht. Dabei gilt Landschaftsökologie vom Gegenstand, Massstab und Inhalt her als prädestiniert anwendungsorientiertes Fach (siehe dazu FINKE 1994). Die Grenzen zwischen Forschung und Anwendung sind auf jeden Fall äusserst unscharf. Der Unterschied zwischen Landschaftsökologie als Forschungsfeld und Angewandter Landschaftsökologie lässt sich also inhaltlich kaum festmachen. Auf der anderen Seite gibt es natürlich eine Lücke zwischen dem, was Wissenschaftler als anwendungsorientiert betrachten und dem, was in der Praxis tatsächlich angewendet wird. Da muss also ein Weg liegen zwischen Forschung und Praxis. Und genau hier liegt der Unterschied! Angewandte Landschaftsökologie geht diesen Weg. Sie umfasst deshalb wesentlich mehr als Forschung und auch mehr als Forschung und Entwicklung. Sie kümmert sich um die Inhalte, Modelle, Verfahren, Konzepte usw. bis sie in die Praxis Eingang gefunden haben. Dies umfasst viele Schritte. Abb. 1 fasst diese Schritte, die sich in die Phasen Grundlagenforschung, Forschung und Entwicklung und Umsetzung gliedern lassen, zusammen.

Es ist eine Binsenwahrheit: Der Weg von der Forschung in die Praxis ist meist langwierig und mitunter mühsam. Ein Beispiel aus dem eigenen Erfahrungsfeld soll dies verdeutlichen. 1999 gab das Niedersä̈HSISCHE LANDESAMT FÜR ÖKOLOGIE das Handbuch «Schutzgut Klima/Luft in der Landschaftsplanung» heraus (NLÖ 1999). Dieses Handbuch ist für die Bearbeitung des Schutzgutes Klima/Luft in der Landschaftsrahmenplanung (Ebene der Landkreise) verbindliche Richtlinie. Es legt das prinzipielle Vorgehen und die Methoden fest. Der Weg seiner Entstehung lässt sich wie folgt zusammenfassen:
1. Experimentelle Untersuchungen (ab 1991).

2. Neues Konzept für prozessorientierte Karten der klima- und immissionsökologischen Funktionen.

3. Pilotprojekte in mehreren Städten und Methodenentwicklung.

4. Ausarbeitung des Handbuches.

5. Diskussion und Abstimmung mit der zuständigen Landesbehörde sowie weiteren Fachbehörden.

6. Politische Diskussion hinter den Kulissen (Anerkennung als Richtlinie).

7. Letzter didaktischer Schliff: Verständlichkeit für Anwender/innen verschiedener Fachrichtungen.

8. Veröffentlichung durch die Landesbehörde (1999).

Acht Jahre hat der ganze Weg beansprucht, wenn auch mit mehreren Unterbrüchen. Über die ganze Zeit hinweg war eine Beteiligung der Forscher erforderlich. Zum Glück dauert es nicht immer so lange. Eine mehrjährige Umsetzungsphase gehört jedoch zu jeder langfristig wirksamen anwendungsorientierten Arbeit. Wer nach Abschluss einer Forschungsarbeit nicht dran bleibt und einfach zum nächsten Projekt oder Thema übergeht, hat keinen Erfolg in der Angewandten Landschaftsökologie.

\section{Eine Matrix des Arbeitsfeldes der Angewandten Landschaftsökologie}

Ein so weites Arbeitsfeld wie die Landschaftsökologie lässt sich inhaltlich natürlich nicht so leicht gliedern (siehe dazu Leser 1997, zu den aktuellen Forschungsperspektiven und Entwicklungstendenzen: MosimanN 1999). In der geographisch betriebenen Angewandten Landschaftsökologie gibt es aber doch einige grosse Themenbereiche, denen sich die meisten Arbeiten zuordnen lassen:

1. Modellierung, Abschätzung und Bewertung von Prozessen im Landschaftshaushalt

Im Vordergrund stehen hier experimentelle Untersuchungen und Modellentwicklungen zur Abschätzung und Bilanzierung der Bodenerosion, des partikelgebundenen Stofftransportes und des Stofftransportes

Der vorliegende Beitrag war Thema des Symposiums «Anwendung und Perspektiven einer integrativen Wissenschaft” anlässlich des 60. Geburtstages von Prof. Dr. Dr. h.c. HaRTMut LESER, veranstaltet am 2. Dezember 1999 in Basel. 


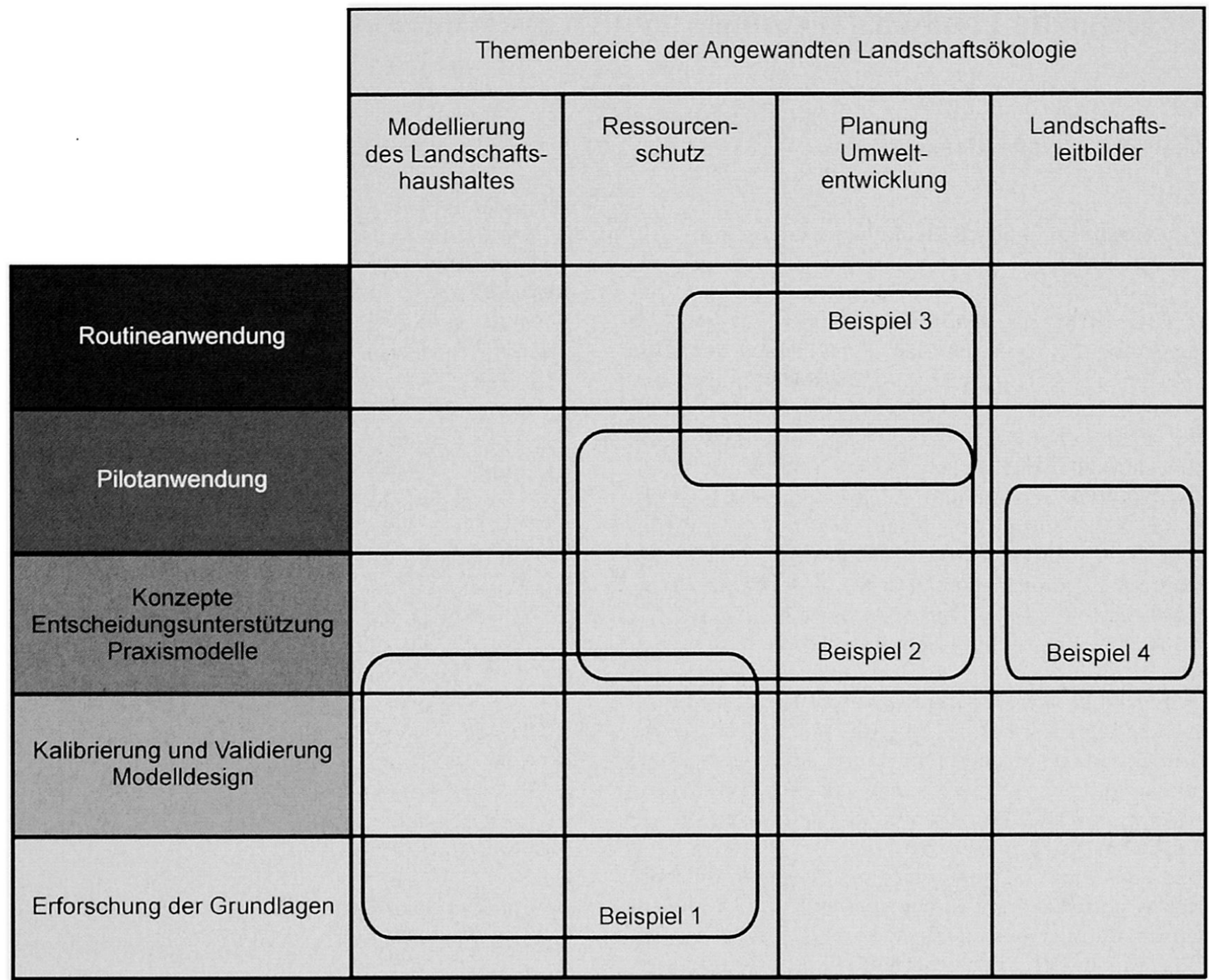

Abb. 1: Matrix des Arbeitsfeldes Angewandter Landschaftsökologie. Die Stellung einer einzelnen Arbeit definiert sich aus dem Forschungs- und Anwendungsbereich und ihrer Einordnung auf dem Weg zwischen Forschung und Praxis.

Activity matrix of applied landscape ecology. The classification of a singular work is defined by the field of research and application and its position on the path between research and practical application.

Matrice du domaine d'activité de l'écologie du paysage appliquée. Un travail est classé selon le domaine de recherche et d'application et selon sa position sur la voie reliant la recherche à la pratique.

vom Boden ins Grundwasser unter Einfluss verschiedener Landnutzungsformen. Wichtig sind weiter die flächendifferenzierte Modellierung von wichtigen Modelleingangsgrössen praxistauglicher Modelle sowie die Erklärung und Prognose von Landschaftsdegradations- und Regenerationsprozessen.

\section{Ressourcenschutz}

Der Themenbereich Ressourcenschutz umfasst von den Grundlagen her die unter Punkt 1 genannten Forschungs- und Entwicklungsarbeiten sowie alle Verfahrensentwicklungen zur Abschätzung und Bewer- tung von Potentialen, Risiken und Funktionen der Schutzgüter Boden, Wasser, Klima/Luft, Flora und Landschaftsbild und zur Tragfähigkeit der verschiedenen Ökosystemtypen. Im weiteren geht es um die Erfassung und Bewertung der Auswirkungen heutiger und zukünftiger Nutzungen auf die natürlichen Ressourcen und die Entwicklung von Konzepten und Massnahmen schonenderer Nutzungsformen.

3. Ökologische Planung und Landschaftsentwicklung Vereinfacht ausgedrückt setzt ökologische Planung und Umweltentwicklung den Ressourcenschutz um. 
Die Angewandte Landschaftsökologie leistet dabei vor allem Beiträge zur Verknüpfung der verschiedenen Umweltbereiche, zur Ermittlung von Vorrangflächen, zur Ermittlung besonders schützenswerter Bereiche (siehe Beispiel 2), zur Ermittlung und Darstellung funktionaler Abhängigkeiten verschiedener Nutzungseinheiten (siehe Beispiel 3) und zur Eingriffsbilanzierung.

Die kurzen Erläuterungen zu den drei Bereichen beanspruchen keine Vollständigkeit, sondern zeigen Hauptperspektiven auf. Die drei genannten grossen Themenbereiche skizzieren einen inhaltlichen Raster für die Angewandte Landschaftsökologie. Dieser inhaltliche Raster lässt sich mit besonderen Themen erweitern (in Abb. 1 der Bereich "Landschaftsleitbilder»). Die Kombination dieser inhaltlichen Gliederung mit den Arbeitsphasen des in Kap. 1 beschriebenen Weges ergeben dann eine Matrix oder ein «System» des ganzen Arbeitsbereiches (Abb. 1). Jede Arbeit in der Angewandten Landschaftsökologie kann gut über ihre Stellung in dieser Matrix eingeordnet, methodisch beurteilt und nach ihrem Grad der Anwendungsreife bewertet werden. Die folgenden vier Beispiele sollen auch dies illustrieren.

\section{Angewandte Landschaftsökologie konkret: vier Bei- spiele angewandter Arbeiten und Ergebnisse}

Genug der Theorie: Ein angewandtes Arbeitsfeld lässt sich mit Beispielen am besten darstellen! Die folgenden Beispiele zu verschiedenen Themen und Phasen auf dem Weg von der Forschung in die Praxis verdeutlichen heutige Modellansätze, Vorgehensweisen, Arbeitsperspektiven und Ergebnisse der Angewandten Landschaftsökologie. Es handelt sich um Beispiele aus dem eigenen Tätigkeitsfeld. Zitate am Schluss jedes Beispiels weisen zusätzlich auf ausgewählte Arbeiten von Kolleginnen und Kollegen im jeweiligen Themenfeld hin.

Eines der wichtigsten Ergebnisse geographischer Arbeit ist und bleibt die digitale Karte. Sie visualisiert räumliche Zusammenhänge auf einer hohen Aggregationsebene und gleichzeitig überschaubar. Dies gilt ganz besonders für die anwendungsorientierte Arbeit, wo Karten eine zentrale Stellung einnehmen für die Vermittlung von Informationen an Fachstellen, Betroffene und Entscheidungsträger. Karten, die aus Kostengründen aber in Schwarz/Weiss-Versionen gedruckt werden müssen, verlieren viel von ihrer Aussagekraft. Deshalb kann die farbige Originalausführung der vier Karten im Internet eingesehen werden (www.geog.uni-hannover.de/phygeo/angewlök).

\subsection{Beispiel 1: Modellierung des Anbindungsgrades} von Ackerflächen an Fliessgewässer

- Einordnung in der Matrix der Angewandten Landschaftsökologie: Modelldesign, Modellkalibrierung und -validierung, Test als Praxismodell.

- Methode/Modell:Wahrscheinlichkeitsmodell auf der Basis logistischer Regression. Die komplexe Grösse "Anbindung" wird von sehr vielen räumlichen Faktoren beeinflusst und lässt sich deshalb nicht «exakt» bestimmen. Das Modell ordnet folglich den Anbindungsgrad auf einer Skala von 0-100\% Anbindungswahrscheinlichkeit ein. Dies geschieht auf Grund einer rasterbasierten Ähnlichkeitsprüfung der die Anbindung beeinflussenden Faktoren mit der Faktorenkonstellation derjenigen Flächen, für die eine Stofflieferung durch Kartierung beobachtet wurde. Das Modell ist bei Voges (1999) ausführlich beschrieben.

- Massstab: 1:25’000 - 1:100’000 (regionale Mesoskale)

- Landschaftsökologischer Ansatz: Reliefeigenschaften, Bodeneigenschaften, Nutzung, Lagebeziehungen, Stofftransportwege und Transportparameter werden zu einer Gesamtgrösse verknüpft. Der Anbindungsgrad liefert somit eine hochaggregierte Aussage über die Schwerpunkte der Herkunft diffuser Stoffeinträge aus dem ganzen Einzugsgebiet.

- Anwendungsbereich: Stoffbilanzierung, regionales Stoffhaushaltsmanagement, Gewässerschutzvollzug (Vollzugsplanung).

\section{Bedeutung des Weges vom kleinen zum grossen Massstab (Downscaling)}

In der Landschaftshaushaltsforschung, Hydrologie, usw. wird die zurzeit intensiv diskutierte Problematik der Regionalisierung grösstenteils als Skalenübergang von "unten nach oben", also vom grossen in den kleinen Massstab gesehen (Upscaling, Aggregierung). Dies ergibt sich aus der Anforderung, die Ergebnisse hochauflösender Prozessforschung und -modellierung in die Fläche zu bringen. In der Praxis liegt dagegen die Sichtweise und die Problematik oft anders. Hochauflösende Daten existieren nur für wenige Flächen. Für ganze Gebiete stellt sich aber die Frage, wo sich die für die Umsetzung von Massnahmen im Umweltschutz relevanten Teilgebiete und Flächen befinden, auf denen Massnahmen dringlich, besonders Erfolg versprechend oder besonders wirksam sind. Hier geht es also um Downscaling. Vereinfachte Modelle, deren Eingangsdaten für ganze Regionen digital verfügbar sind, ermitteln die relevanten Flächen, für die in einem zweiten Schritt ein bis zwei Massstabse- 


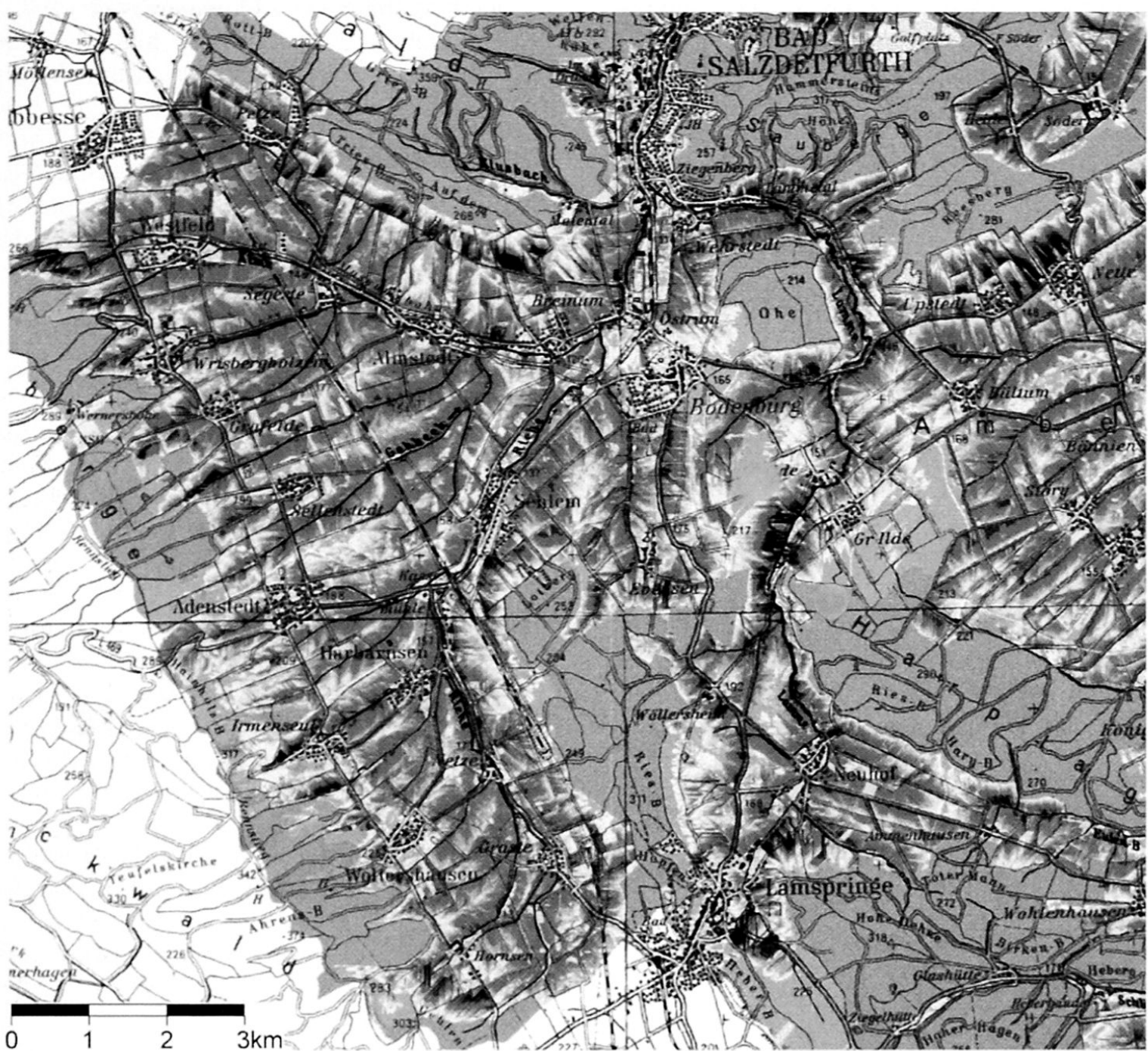

Gewässeranbindungsgrad der aktuellen Situation

Wahrscheinlichkeit des Feststoffeintrags [\%]

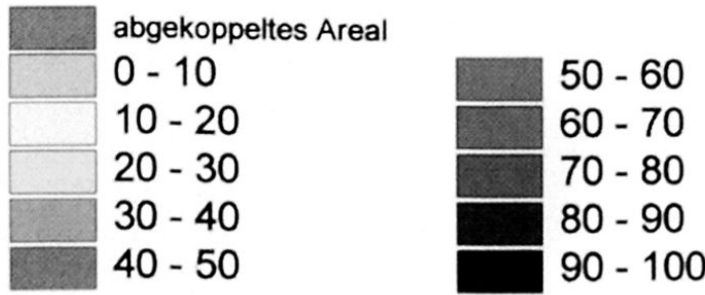

$\square$ Fläche ohne Stofflieferfunktion Siedlung

Gewässer, landwirt. Graben

Abb. 2: Anbindung von Ackerflächen an Fliessgewässer: Modellierung des Anbindungsgrades mit einem Wahrscheinlichkeitsmodell (farbige Originalabbildung siehe: www.geog.uni-hannover.de/phygeo/angewlök/) Linkage of agricultural fields to rivers: modelling degree of interconnectedness by a probability model (original colour map in www.geog.uni-hannover.de/phygeo/angewlök/)

Rattachement des surfaces cultivées aux cours d'eau: modélisation du degré de rattachement à l'aide d'un modèle de probabilité (carte originale en couleurs, voir: www.geog.uni-hannover.de/phygeo/angewlök/)

(Quelle: Voges 1999; Kartographie: V. WickenKamp) 
benen tiefer mit räumlich und eventuell zeitlich höher auflösenden Modellen genauere Abklärungen getroffen werden. Das Verfahren zur Berechnung des Gewässeranbindungsgrades ist ein typischer Vertreter mittelmassstäblicher Modelle mit dem Ziel der Ermittlung von Schwerpunkträumen für genauere Abklärungen und das Setzen von Schwerpunkten für Massnahmen.

\section{Berechnung des Anbindungsgrades}

In die Berechnung des Anbindungsgrades fliessen die Faktoren Gewässerdistanz, Stofftransportpfaddistanz, Direktabflussvolumen, Hangeinzugsgebietsgrösse, Hanglänge, Hangneigung, Hangform, Erosionsgefährdung und Transportkapazität der Gräben ein. Dies sind die Parameter eines multiplen Regressionsmodells, dessen Fitting mit der Maximum-Likelihood-Methode erfolgt (korrekte Ermittlung der höchstmöglichen Zahl beobachteter Lieferflächen). Die Regression ergibt den Exponenten $\mathrm{z}$ in der Gleichung

\section{1 \\ $\mathrm{pA}$ (Anbindungsgrad) $=\frac{1}{1+\mathrm{e}^{\mathrm{z}}}$}

(Hosmer \& Lemeshow 1989).

Der berechnete Anbindungsgrad lässt sich klassifizieren (Abb. 2) oder über einen Schwellenwert der Anbindungswahrscheinlichkeit (z.B. 50\%) in die Aussage «Fläche angebunden» / «Fläche nicht angebunden» transformieren.

\section{Bemerkungen zum Kartenbeispiel}

Die Karte stellt einen Ausschnitt aus dem Einzugsgebiet der Innerste in Niedersachsen dar. Das gesamte modellierte Gebiet ist $500 \mathrm{~km}^{2}$ gross. Das Kartenbild basiert auf einer Modellierung im $25 \mathrm{~m}$-Raster. Durch die feine Wahrscheinlichkeitsklassierung in 10\%-Schritten ergeben sich vom optischen Eindruck her unscharfe Übergänge, eine gewollte Annäherung an den Kontinuumscharakter aller prozessualen Phänomene.

Etwa die Hälfte der Gebietsfläche liefert diffuse Stoffausträge in die Gewässer. Der Anbindungsgrad zeigt eine kleinräumige Differenzierung. Flächen mit mittlerer bis hoher Stofflieferwahrscheinlichkeit scharen sich vor allem um das Gewässer- und Grabensystem, weil die Gewässerdistanz und Stofftransportpfaddistanz eine wichtige Rolle spielen. Bei hoher Erosionsgefährdung oder starker Begünstigung von gebündeltem Abfluss in Tiefenlinien können mit hoher Wahrscheinlichkeit stoffliefernde Flächen aber auch bis $700 \mathrm{~m}$ vom nächsten Vorfluter entfernt liegen.

Bei etwa $30 \%$ der Landwirtschaftsfläche ist die
Wahrscheinlichkeit der Anbindung $>50 \%$. Dies sind die hauptsächlich diffuse Stoffausträge liefernden Flächen und somit der Massnahmenschwerpunkt für Erosionsbekämpfung und Abflussregulierung. Diese Flächen befinden sich viel häufiger im Bereich von Gräben als von permanenten Fliessgewässern. Die Auenbereiche der Flüsse wie Nette und Lamme weisen sogar häufig reduzierte Eintragswahrscheinlichkeiten auf. Wegen der geringen Hangneigung sind die Feststoffeinträge durch Bodenerosion hier unwahrscheinlich. Nicht berücksichtigt bleiben dabei aber Stoffein- und -austräge durch rückströmendes Wasser bei Überschwemmung. Durch Schliessung der Gräben liessen sich $40 \%$ der Landwirtschaftsfläche von den Gewässern abkoppeln. Der für eine solche effiziente Massnahme notwendige Flächenbedarf wird zurzeit in einer gesonderten Untersuchung bilanziert.

Wie gut ist ein solches Wahrscheinlichkeitsmodell? Die Überprüfung in der untersuchten Region auf der Basis der kartierten Gebiete ergibt einen maximalen Anteil der richtig vorhergesagten Flächen (angebunden und nicht angebunden) von 75\% (Voges 1999). Für andere Arbeiten zum Thema siehe: BaCH et. al. (1994), Prasuhn \& Braun (1995), Grunwald \& Frede (1999), Young et al. (1987), Bronstert et al. (1998).

\subsection{Beispiel 2: Ausscheidung besonders schutz-} würdiger Bodenflächen für die Raumplanung

- Einordnung in der Matrix der Angewandten Landschaftsökologie: Konzept und Praxismodell sowie Pilotanwendung im Bereich Ressourcenschutz und Planung.

- Methode/Modell:Algorithmen in Form von Entscheidungsleitern ermitteln die besonders schützenswerten Bodenflächen für sieben Bodenfunktionen. Die wichtigsten Eingangsdaten sind flächendeckende Bodendaten im Massstab 1:5'000. Konzept und Methode sind bei MosimanN (1999) ausführlich erläutert.

- Massstab: 1: 5’000 - 1:25’000

- Landschaftsökologischer Ansatz: Der Boden wird umfassend betrachtet und mit Standorteigenschaften (Relief, Gestein, Wasserhaushalt), Nutzungseignung, Schutzansprüchen und Immissionssituation verknüpft. Die Eignungsbewertung erfolgt direkt verbunden mit der Einschätzung von Risiken.

- Anwendungsbereich: Landschaftsplanung, Landschaftsentwicklungskonzepte, Regionalplanung, Richtplanung, Nutzungsplanung.

Die Karte der besonders schützenswerten Bodenflächen kann ein Scharnier zwischen Bodenschutz und Raumplanung bilden. Sie steht in einer Schlüssel- 


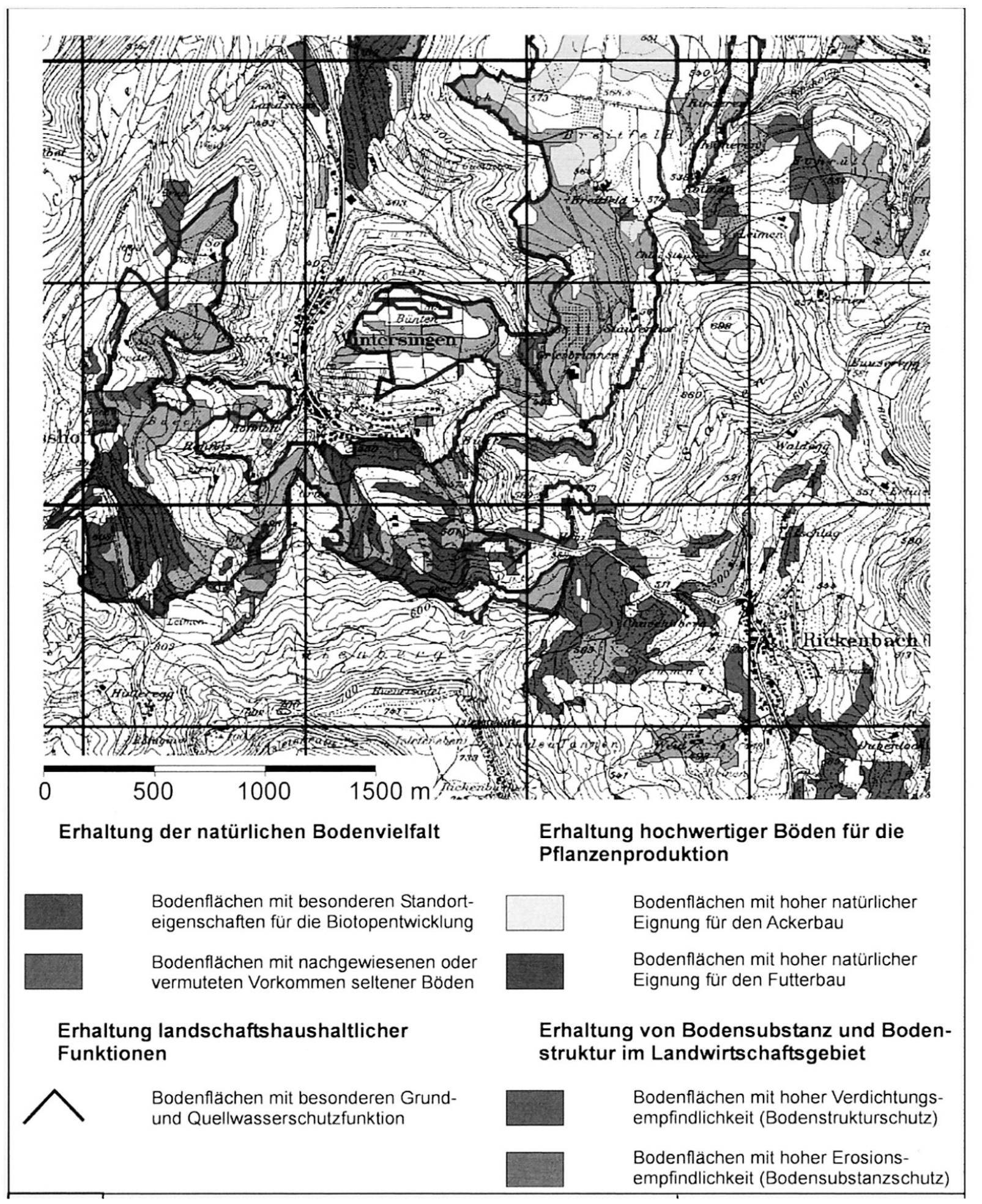

Abb. 3: Inventar besonders schutzwürdiger Bodenflächen für die Raumplanung. Erstellt nach der Methode von Mosimann (1999) im Auftrag des Amtes für Umweltschutz und Energie des Kantons Basel-Landschaft (farbige Originalabbildung siehe www.geog.uni-hannover.de/phygeo/angewlök/)

Inventory of soils of special conservation value for land use planning. Evaluated by the method described in MosiMANN (1999), for the Department for Environmental Conservation and Energy, Canton "Basel-Landschaft» (original colour map in www.geog.uni-hannover.de/phygeolangewlök/)

Inventaire des sols méritant une protection particulière pour l'aménagement du territoire. Elaboré d'après la méthode de Mosimann (1999), à la demande de l'Office de la protection de l'environnement et de l'énergie du canton de Bâle-Campagne (carte originale en couleurs, voir: www.geog.uni-hannover.de/phygeo/angewlök/) 
funktion für den Transfer der Bodenschutzanliegen in die Planung. Sie formuliert die fachlichen und flächenmässigen Anforderungen des Bodenschutzes an die Raumplanung. Dabei stellt sie die Schutzanliegen zusammengefasst und in aufbereiteter Form kartografisch dar, unter Berücksichtigung von Funktionen, Eignungen, Risiken und besonderen Werten des Bodens (Schutz seltener und regionstypischer Böden, Schutz von Böden mit besonderer Eignung als Standort für Kulturpflanzen oder naturnahe Pflanzengemeinschaften (Biotopentwicklungspotential), Schutz der Filterfunktion des Bodens, Vermeiden belastender Nutzungen auf empfindlichen Böden). In der Karte werden nur die besonders schützenswürdigen Bodenflächen ausgewiesen, damit ihr im raumplanerischen Abwägungsprozess auch das entsprechende Gewicht zukommt. In diesem Sinne zeigt die Karte namentlich

- welche Bodenflächen besonderen Schutz benötigen,

- wo sich schützenswerte Bodenobjekte (seltene Böden) befinden könnten,

- wo besondere Risiken einer schleichenden Bodenzerstörung bestehen,

- wo sich besonders ertragreiche Böden befinden,

- wo sich für die Biotopentwicklung besonders wichtige Böden befinden und

- wo Aspekte des Bodens in die allgemeine Begründung von Schutzflächen einfliessen können.

Die Karte der schutzwürdigen Bodenflächen beruht auf folgendem Konzept:

- Bestimmung der Schutzwürdigkeit der Böden auf der Grundlage von definierten, aus der Bodenkarte 1:5'000 ableitbaren Einzelmerkmalen des Bodens. Dadurch ist es möglich, nachvollziehbare Standards der Schutzwürdigkeit festzulegen. Zudem kann mit den gleichen Grundlagen auf verschiedenen Massstabsebenen gearbeitet werden, also in der regionalen und kommunalen Planung.

- Betrachtung des Bodens in seinen standörtlichen Rahmenbedingungen. Die Schutzwürdigkeit einer Bodenfläche hängt nicht nur von den Merkmalen des Bodens selbst, sondern je nach Einzelaspekt auch von Relief, Lokalklima oder Gestein ab.

- Verknüpfung von Potentialen und Risiken: Schutzwürdigkeit ist nur gegeben, wenn beispielsweise eine Fläche mit hoher natürlicher Eignung für die Pflanzenproduktion nicht gleichzeitig besonders erosionsoder verdichtungsanfällig ist.

Die Standards legen fest, welche Böden im Hinblick auf die Erhaltung von Bodenflächen besonders schützenswert und welche im Hinblick auf die Vermeidung von Belastungen besonders empfindlich sind. Bodenflächen, welche die Standards und definierten Schwellenwerte erfüllen, gelten als schützenswert und werden in der Karte der schützenswerten Bodenflächen ausgewiesen. Die Standards sind in Mosimann (1999) erläutert.

Die schutzwürdigen Bodenflächen können auf mehreren Wegen in die Raumplanung integriert werden:

- Festlegung von Vorranggebieten für den Bodenschutz.

- Einbezug des Bodenschutzes in andere Schutzgebiete, was auch eine Verbreiterung der ökologischen Argumentationsbasis bedeutet.

- Bezeichnung der für die Landwirtschaft besonders wichtigen Böden.

- Differenzierung des Landwirtschaftsgebietes unter dem Aspekt des Bodenschutzes in Intensiv- und Extensivgebiete.

Andere Arbeiten zum Thema: Bauer et al. (1997), SCHRAPS \& SCHREY (1997), ARL (1999).

\subsection{Beispiel 3: Von der synthetischen Karte der klima- und immissionsökologischen Funktionen zur Planungskarte}

- Einordnung in der Matrix der Angewandten Landschaftsökologie: Routineanwendung für die Flächennutzungsplanung.

- Methode/Modell: Typisches Beispiel für einen Methodenkomplex aus Messung, empirisch-statistischer Modellierung, ökologischer Bewertung und Synthese. Das methodische Vorgehen ist bei MosiMANN et al. (1999) zusammengefasst.

- Massstab: 1:5’000 - 1:50’000

- Landschaftsökologischer Ansatz: Es werden lufthaushaltliche Abhängigkeiten zwischen Stadt und Umland unter funktionaler Verknüpfung klimatischer und lufthygienischer Aspekte betrachtet. Entscheidend sind Wechselwirkungen zwischen Oberflächenstruktur und Strömung und die nutzungsabhängige Differenzierung der Luftbelastung im Ausgleichs- und Wirkungsraum. Auf der Basis verschiedener Methoden wird eine Zusammenschau der funktionalen Abhängigkeiten und Bedeutung der Teilräume erarbeitet und in eine Karte mit Aussagen zu Handlungsschwerpunkten transformiert.

- Anwendungsbereich: Stadtplanung, Landschaftsplanung.

\section{Synthese der klima- und immissionsökologischen Funktionen}

Die Darstellung der klima- und immissionsökologi- 


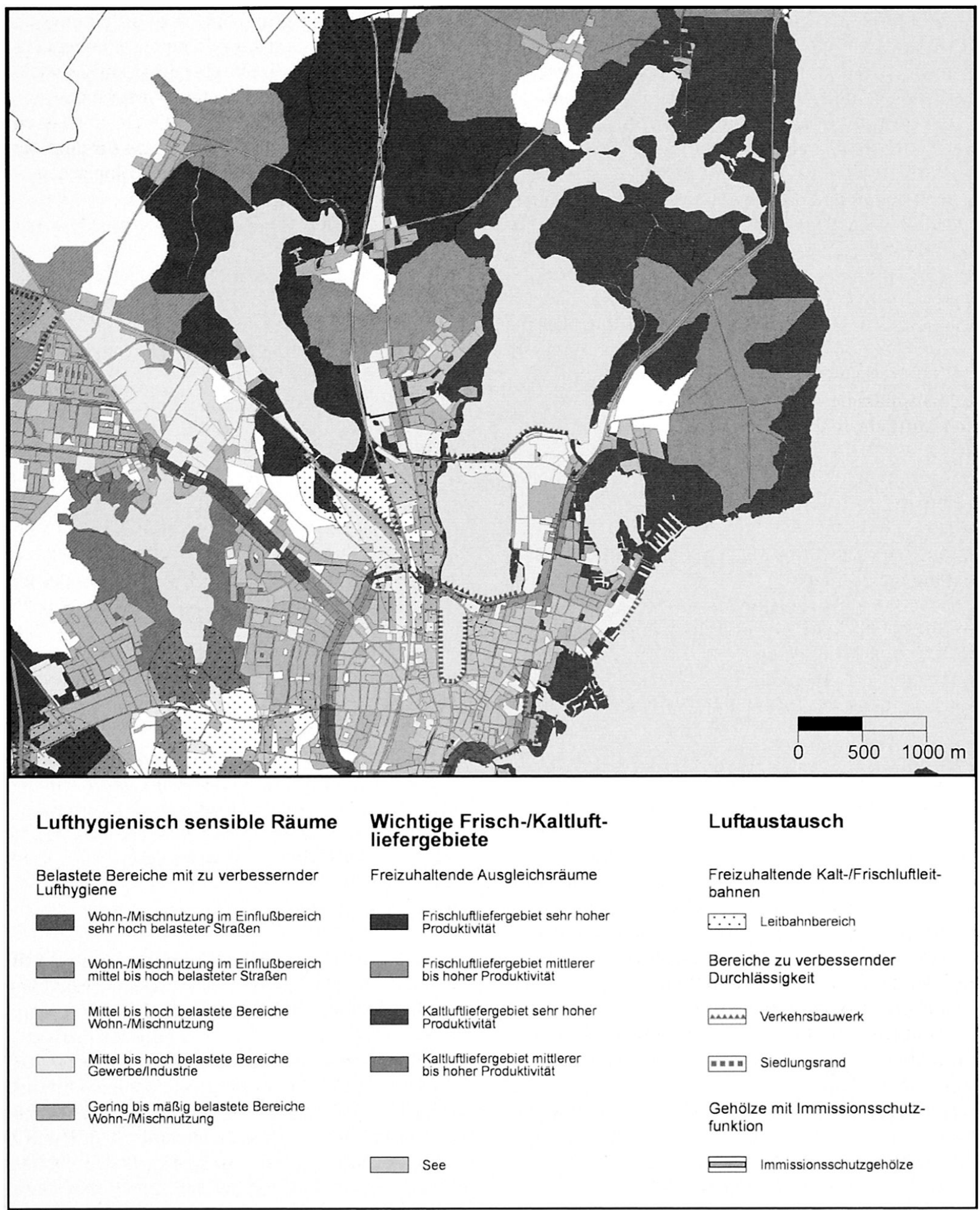

Abb. 4: Klima- und immissionsökologische Planungskarte für die Landeshauptstadt Schwerin (farbige Originalabbildung siehe www.geog.uni-hannover.de/phygeo/angewlök/)

Map showing planning proposals for the improvement of climate and air quality in the State capital of Schwerin (original colour map in www.geog.uni-hannover.de/phygeo/angewlök/)

Carte du climat et des immissions pour le chef-lieu Schwerin (carte originale en couleurs, voir: www.geog.unihannover.de/phygeo/angewlök/)

(Quelle: Mosimann et al. 1999; Kartographie: V. WickEnKamp) 
schen Funktionen erfolgt in synthetischen Karten, die den planungsrelevanten Ist-Zustand der Belastung und der Austauschfunktionen wiedergeben (Belastungsdifferenzierung des Ausgleichs-Wirkungsraumgefüges und der vermittelnden Luftaustauschprozesse). Dies ergibt in der Karte die drei Elementgruppen lufthygienische Situation, Kalt-/Frischluftproduktion in den Ausgleichsräumen und Luftaustauschsysteme. Im Einzelnen enthalten Karten der klima- und immissionsökologischen Funktionen folgende Elemente:

- Ausgleichs- und Wirkungsräume allgemein,

- nach Luftbelastung klassifizierte Wirkungsräume (generell),

- nach Überwärmung klassifizierte Wirkungsräume (je nach Ausmass der Überwärmung),

- nach Luftbelastung klassifizierte Hauptstrassen,

- nach Kaltluftproduktivität klassifizierte Kalt-/Frischlufteinzugsgebiete,

- Kaltluftsammelgebiete,

- übergeordnete, für den Austausch wichtige Winde,

- thermisch und/oder orographisch bedingte Ausgleichsströmungen (klassifiziert nach Massenströmungen),

- Reichweiten/Wirkungsbereiche von Kalt-/Frischluft,

- zwischen Ausgleichs- und Wirkungsraum vermittelnde Strukturen/Flächen (vor allem Leitbahnen) und

- Austauschhindernisse (strömungsbehindernde Strukturelemente).

Der Gesamtumfang der dargestellten Inhalte und der Detaillierungsgrad der einzelnen Informationen hängt von der lokalen Problemlage und der Bearbeitungstiefe ab. Der vom Auftraggeber geforderte Betrachtungsmassstab beeinflusst die darzustellende räumliche Auflösung sowie die Grössenordnung und Untergrenzen der abgebildeten Elemente, Prozesse und Funktionszusammenhänge. Karten der klima- und immissionsökologischen Funktionen sind also trotz der prinzipiell immer gleichen Inhalte individuell auf den jeweiligen Planungsraum zugeschnitten. Entscheidend ist aber in jedem Fall, dass

- Ausgleichs-Wirkungsraum-Beziehungen sichtbar werden,

- Wirkungszusammenhänge darstellerisch dominieren,

- Belastungen klassifiziert werden und

- nur (planerisch) wesentliche Funktionsbeziehungen dargestellt werden.

\section{Abgeleitete Planungskarten}

Aus der differenzierten Analyse und Darstellung der klima- und immissionsökologischen Situation lassen sich generelle raum- und problembezogene Planungsempfehlungen für die Schutzgüter Klima und Luft ableiten. Die Planungskarte soll die relevanten Informatio- nen als Abwägungsgrundlage in den Planungsprozess einbringen. Ihr kommt somit eine entscheidende Rolle als Mittler zwischen klimatisch-lufthygienisch wichtigen Belangen und den planerischen Ansprüchen zu (vgl. VDI-Richtlinie 3787 Bl. 1 1994). Gleichzeitig lassen sich mit dieser Karte Handlungsprioritäten für eine Verbesserung der klima- und immissionsökologischen Situation im Untersuchungsraum festlegen. Man kann mindestens zwei Arten von Planungskarten unterscheiden:

1. Karten mit wichtigen Elementen aus dem Komplex Klima/Luft, an die konkrete Handlungsempfehlungen gebunden sind, oder

2. stärker generalisierte Karten, die eine klima- und immissionsökologisch verträgliche Sollstruktur für ein Plangebiet darstellen.

Letztere beinhalten übergeordnete Planungsziele. Es geht dabei um die Ausweisung von Vorrangflächen für die Erhaltung und die Entwicklung klima- und/oder immissionsökologisch wichtiger Freiflächen, die bei der zukünftigen Siedlungs- und Freiflächenentwicklung möglichst von einer Bebauung freizuhalten sind. Solche Karten richten sich vor allem an Entscheidungsträger in der Verwaltung, an Politiker und interessierte Bürger. Die erstgenannten Karten mit Handlungsempfehlungen sind dagegen konkreter und richten sich gezielter an die Planenden. Dies lässt sich mit der «Karte zu den Planungsempfehlungen zu den Schutzgütern Klima und Luft» für die Landeshauptstadt Schwerin (Abb. 4) verdeutlichen. Mit dieser Karte ging es vor allem darum,

- begrenzt auftretende Belastungen zu lokalisieren und hinsichtlich des Belastungspotentials zu bewerten. Daraus lassen sich nach Priorität geordnete Handlungsempfehlungen ableiten.

- Hinweise zu Vorrangflächen für die Frisch-/Kaltluftproduktion und zur Optimierung des autochthonen Luftaustausches zu geben.

Die Planungskarte dient gleichzeitig dazu, im Rahmen der Flächennutzungsplanung Konflikte mit konkurrierenden Planungen (Wohngebietsausweisungen, Industrieansiedlungen, Verkehrsplanungen) aufzuzeigen. Sie bezieht sich dabei vorrangig auf die Luftaustauschprozesse zwischen der Schweriner Kernstadt und den Freiflächen im Umland, die für diese lufthygienisch belasteten Areale eine relevante Ausgleichsleistung erbringen können.

In den Planungskarten sind die Funktionen grundsätzlich wirkungsraumbezogen, handlungs- und damit umsetzungsorientiert dargestellt. Der Nutzen von aus Klimafunktionskarten abgeleiteten Massnahmen ist damit von den Funktionen und dem Problemstand her nachvollziehbar. Dieses Instrument eignet sich somit 
für die räumliche Planung wie auch für vorhabensbezogene Umweltuntersuchungen, wie Umweltverträglichkeitsprüfungen. Besondere Relevanz kommt den Karten aber in der Stadtplanung mit ihrem hohen Problemdruck zu. Für andere Arbeiten zum Thema siehe: ZimmermanN (1988), Reuter et al. (1991), VDI (1994), Sтоск(1992).

\subsection{Beispiel 4: Ableitung prozessorientierter land- schaftsökologischer Leitbilder für Multifunktions- landschaften}

- Einordnung in der Matrix der Angewandten Landschaftsökologie: Modellkonzept, Praxismodell, Modelltest.

- Methode/Modell: Wissensbasierte Aggregationsmethode auf hoher Ebene. Formalisierung des Wissens in Form von landschaftsspezifischen Standards, einer hierarchischen Entscheidungsleiter und Verknüpfungsmatrices. Die standardisierte Methode liefert einen Grundraster landschaftsökologisch optimierter Verteilung von Nutzungs- und Schutzflächen. Die hohe Syntheseebene verlangt jedoch eine gebietsindividuelle Nachbearbeitung. Die Methode ist in Mosimann et al. (2001) ausführlich dargestellt.

- Massstab: 1:10'000 - 1:50'000. Für die Ermittlung übertragbarer Werte der Nutz- und Schutzflächenanteile müssen Gebiete von mindestens $15 \mathrm{~km}^{2}$ Grösse bearbeitet werden.

- Landschaftsökologischer Ansatz: Verständnis des landschaftlichen Mosaiks als ein Arealmuster aus haushaltlich-funktionalen Prozesseinheiten mit Funktionsüberlagerungen und lateralen Abhängigkeiten. Umfassende Verknüpfung von bis zu 20 Einzelfunktionen. Gesamtbewertung durch Ist-Soll-Bilanz der Anteile der verschiedenen Nutzungs- und Schutzflächen.

- Anwendungsbereich: Landschaftsplanung, Landschaftsentwicklungskonzepte, Regionalmanagement, akteurorientierte Planungsprozesse, Agenda 21Prozesse (ökologische Nachhaltigkeitsprüfung).

\section{Die landschaftsökologischen Funktionskategorien}

Aufbauend auf dem heutigen haushaltlichen Verständnis einer Landschaft geschieht die Funktionsverknüpfung und -überlagerung für die Ableitung eines prozessorientierten Leitbildes in vier Funktionskategorien (Zuordnung der Einzelfunktionen siehe Abb. 5).

1. Nutzungsfunktionen:Die Nutzungen im nicht bebauten Raum, die einem Areal auf Grund seiner natürlichen Eignung zugewiesen werden können.

2. Schutzfunktionen: Fähigkeiten und Aufgaben von
Arealen zur Entwicklung und Beherbergung einer natürlichen Lebensgemeinschaft, zur Entwicklung eines naturbestimmten Standort- bzw. Ökotopmusters mit der entsprechenden «Prozessdynamik» zur Erhaltung von Böden sowie zur Bewahrung von Formen und Erscheinungen der unbelebten Natur oder der früheren Kulturlandschaft.

3. Regulationsfunktionen: Fähigkeiten und Aufgaben von Arealen zur ausgleichenden Steuerung natürlicher Prozesse, zur Rückhaltung und Transformation schädlicher Stoffe, zum Abbau ökologischer Belastungen und zur Verbesserung der Funktionsfähigkeit aller Teilsysteme der Landschaft.

4. Pufferfunktionen: Aufgaben von Arealen zur Verhinderung bzw. Verminderung schädlicher Einwirkungen auf Schutzflächen und empfindliche Nutzung und zur Abschottung von Arealen mit hohen Emissionen.

Die meisten Areale einer ökologisch optimierten Landschaft können Funktionen aus mehreren Kategorien übernehmen. Die Regulations- und Pufferfunktionen stehen dabei nie selbständig, sondern sind immer an Nutzungs- und/oder Schutzfunktionen angebunden. Das Arealmuster eines Leitbildes besteht deshalb ausschliesslich aus Nutzungs- und Schutzgebieten, hinter denen die anderen beiden Funktionen stehen und auf den Karten z.T. auch zusätzlich abgebildet werden.

\section{Modellaufbau}

Die wissensbasierte Ableitung eines funktional begründeten landschaftsökologischen Leitbildes ist ein induktiver Prozess, der über viele Schritte läuft. Im Zentrum stehen ein hierarchisches Verknüpfungsmodell, eine Matrix für die Funktionsüberlagerung und eine Matrix zur Ermittlung der Pufferräume. Die Ableitung gliedert sich im Wesentlichen in vier Teile:

1. Schutzgebiete, Schutzfunktionen: Hier werden alle für den Totalschutz, den Arten-, Biotop- und Geotopschutz und den Prozessschutz notwendigen Flächen herausgefiltert. Gleichzeitig ermittelt dieser Teil die auf den einzelnen Schutzflächen zulässigen Nutzungen.

2. Regulationsfunktionen: Hier werden für die Flächen mit Regulationsfunktionen über Schlaufen in die Matrix «Funktionsüberlagerung» ebenfalls die zulässigen Nutzungen ermittelt.

3. Nutzungsoptimierung: Hier erfolgt die Zuordnung von Acker- und Grünlandnutzung und die Nutzungsintensität auf der Grundlage der Standorts eignung und der Risiken für Boden und Gewässer.

4. Ermittlung der Pufferraumfunktionen und der erforderlichen Pufferräume (Matrix zum Pufferraumbedarf). 


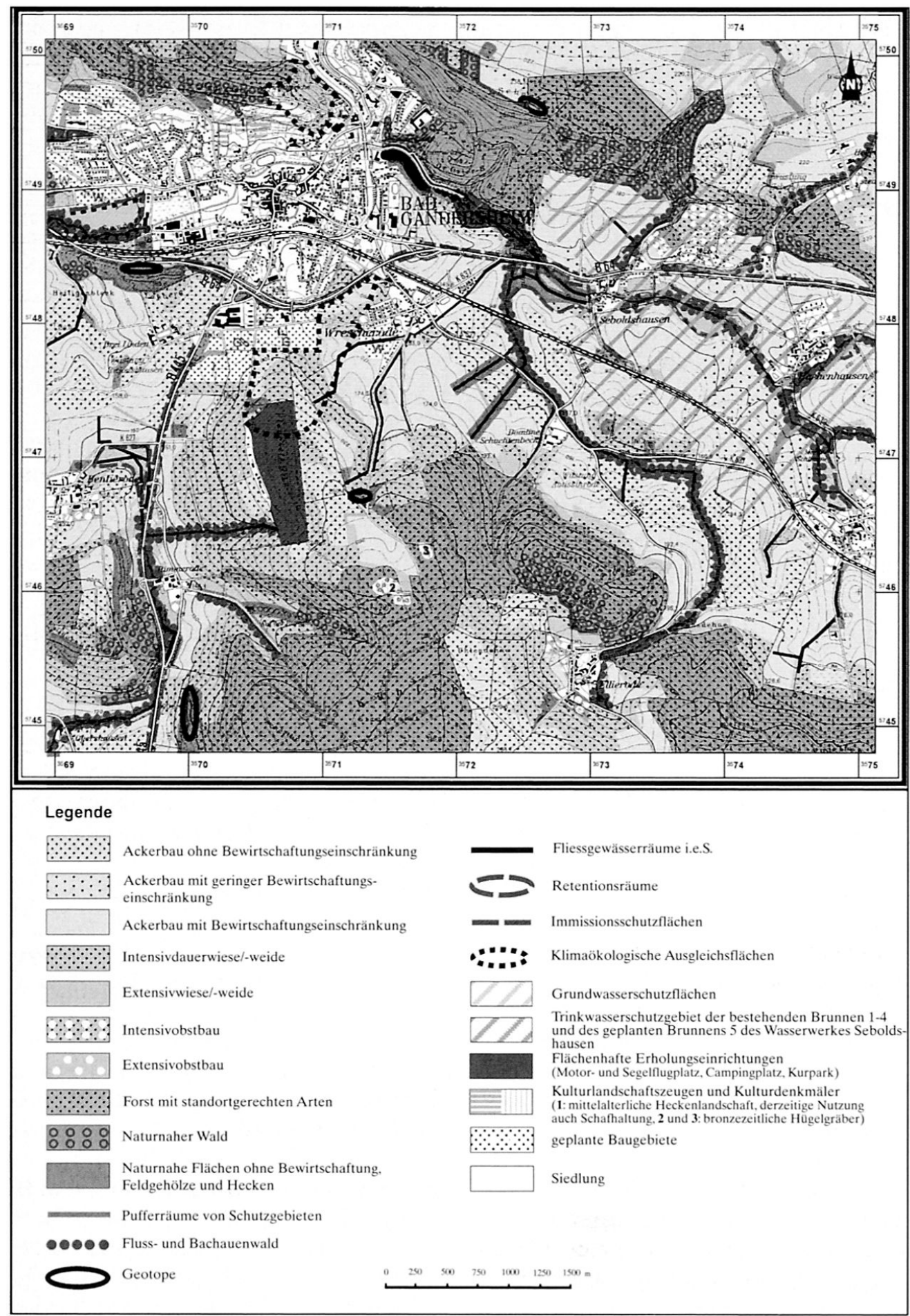

Abb. 5: Funktional begründetes landschaftsökologisches Leitbild für den Landschaftstyp des lössbedeckten Berg- und Hügellandes: Beispiel Leinebergland bei Bad Gandersheim (Südniedersachsen) (farbige Originalabbildung siehe www.geog.uni-hannover.de/phygeo/angewlök/)

Landscape development concept based on ecological functions of a mountain and hilly terrain covered by loess. The example is taken from "Leinebergland" near Bad Gandersheim (southern Lower Saxony), (original colour map in www.geog.uni-hannover.de/phygeo/angewlök/)

Concept fonctionnel pour le paysage d'un pays de montagnes et de collines couvert de loess: l'exemple du "Leinebergland» près de Bad Gandersheim (Basse-Saxe méridionale) (carte originale en couleurs, voir: www.geog.unihannover.de/phygeo/angewlök/)

(Quelle: Mosimann et al. (2001); Kartengrundlage: Topographische Karte 1:25’000, Blatt 4126, Bad Gandersheim; Kartographie: I. KÖHLER) 
Die zu durchlaufenden Arbeitsschritte münden in ein mehrschichtiges Ergebnis. Dies besteht im Wesentlichen aus drei Teilen:

1. Einer Karte des Soll-Zustandes der Landnutzung (dem Leitbild im engeren Sinne).

2. Den Kennzahlen für die optimalen bzw. vertretbaren Anteile der verschiedenen Nutzungs- und Schutzflächen. Diese spezifischen Flächenanteile stellen eine Bilanz des Soll-Zustandes dar. Im Rahmen noch weiter zu prüfender Grenzen besitzen diese Kennzahlen Gültigkeit für den untersuchten Landschaftstyp, sofern das untersuchte Gebiet genügend gross gewählt wurde.

3. Der Bewertung des aktuellen Grades der ökologischen Anpassung der Nutzung. Dies geschieht durch den Vergleich Ist-Zustand/Soll-Zustand der Landnutzung. Mit einer solchen Bilanz lässt sich auch jede neue Planung messen, z.B. mit der Frage, wie weit sich die geplante Entwicklung in Richtung Soll-Zustand bewegt.

\section{Bemerkungen zum Leitbildbeispiel (Abb. 5)}

Das vorgestellte Beispiel liegt im südwestlichen Harzvorland im Leinebergland (Südniedersachsen). Es handelt sich um ein typisches Ackerbaugebiet, bedingt durch die überwiegende Lössbedeckung der mesozoischen Schichten.

Auch in einer landschaftsökologisch optimierten Landschaftsstruktur bleiben im lössbedeckten Bergland die Ackerflächen prägend. Diese müssen jedoch in unterschiedliche Bewirtschaftungsintensitäten differenziert werden. Die Einzelflächen für intensiven Ackerbau ohne Einschränkungen liegen dabei innerhalb grösserer Flächen, für die eine besonders bodenschonende Bewirtschaftung erforderlich ist. Diese generell intensiv nutzbaren Flächen erreichen dabei im Einzelfall maximale Ausdehnungen von ca. 50 ha. Die Wiesen liegen überwiegend im Ackerbaugebiet eingestreut. Intensivwiesen müssten lediglich auf den steileren Hängen im südwestlichen Teil grössere Flächen einnehmen. Die Extensivwiesen konzentrieren sich überwiegend in Säumen längs der grösseren Bäche und an den Waldrändern, eine Grundanordnung, die dem früheren Landschaftszustand recht nahe kommt. Die Extensivwiesen tragen damit zusammen mit den Fluss- und Buchauenwäldern viel zur Strukturierung der Ackerbauflächen bei. Die flächenmässig wichtigsten, das Muster der Nutzungs- und Schutzflächen bestimmenden Regulationsfunktionen sind der auszuweitende Trinkwasserschutz, der Grundwasserschutz und die Hochwasserretention. Klimaökologische Ausgleichsflächen spielen wegen der geringen Grösse der Stadt Bad Gandersheim nur eine untergeordnete Rolle. Die Stoffretention verbessert sich durch die Aus- weitung der Fliessgewässerräume, eine konsequente bodenschonende Bewirtschaftung (Kategorie «Ackerbau mit Bewirtschaftungseinschränkungen») und durch zusätzliche Pufferstreifen längs der Gräben.

Das funktional abgeleitete landschaftsökologische Leitbild weicht stark vom bestehenden Zustand ab. Die wichtigsten Unterschiede zwischen heutiger Landnutzung und Landschaftsstruktur und Leitbild sind: Umwandlung eines Teils der Ackerflächen in Intensivwiesen, die Wiedereinführung von Extensivwiesen als Nutzungselement im gesamten Gebiet, die Differenzierung des Ackerbaugebietes in drei Bewirtschaftungsintensitäten, die Umwandlung von Forstflächen in Wirtschaftswälder mit standortgerechten Baumarten, die Ausweitung naturnaher Waldflächen und die generelle Schaffung von Räumen für eine naturnahe Entwicklung aller Fliessgewässer.

Andere Arbeiten zum Thema: BASTIAN \& RöDER (1996), Wiegleb (1997), Deutscher Rat Für LandesPFLEGE (1997).

\section{Ein Blick in die Zukunft}

Die vier Beispiele stehen stellvertretend für andere. Sie decken thematisch nur einen begrenzten Bereich der extrem vielfältigen Arbeitsperspektive «Landschaftsökologie» ab. Trotzdem enthalten sie die meisten grundlegenden Elemente heutiger und zukünftiger Arbeit in der Angewandten Landschaftsökologie. Welches sind diese grundlegenden Elemente?

\section{Funktionen und Prozesse}

Sie stehen heute im Vordergrund jeder Landschaftsanalyse. Die Vielfalt der verfügbaren Simulations- und Schätzmodelle erlaubt heute in Forschung und praxisorientierter Arbeit flächendifferenzierte Analysen der wichtigsten landschaftshaushaltlichen Zustände und Prozesse. Dies gilt vor allem für den grossen Massstab (Parzelle und elementare Einzugsgebiete). In der Einzugsgebietsmesoskale und in der regionalen Mesoskale bestehen nach wie vor grössere Lücken. Die Landschaftsökologie sollte sich in Zukunft in der Modellierung vermehrt in diesem sog. mittleren Massstab bewegen und die regionale Übertragbarkeit der Ergebnisse verbessern. Generell muss zudem gerade heute immer wieder daran erinnert werden, Modellierung nicht isoliert zu betreiben, sondern eingebunden in Beobachtung. Funktionen und Prozesse lassen sich in räumlicher Differenzierung nur im Methodenverbund genügend sicher erfassen und prognostizieren.

2. Aussagesicherheit und Wahrscheinlichkeit

Alle wissen (unterdessen), dass es die der Struktur- 
beschreibung und Flächenmodellierung unterstellte Homogenität der Elementarflächen nicht gibt und die Komplexität der Systeme nie eindeutige Aussagen erlaubt. Auch die Praxis erwartet in Zukunft vielmehr als früher Angaben zur Sicherheit der gelieferten Aussagen. Das Beispiel «Anbindungsgrad von Ackerflächen an Fliessgewässer» geht in diese Richtung, indem es die "weiche» Grösse Gewässeranbindung auf einer Wahrscheinlichkeitsskala quantifiziert. Noch grundlegender ist aber ein in Zukunft anderer Umgang mit der Heterogenität aller landschaftsökologischer Raumeigenschaften. Hier müssen Modelle entwickelt werden, die Datenfelder bekannter Heterogenität generieren. Auf dieser Basis kann der datenabhängige Schätzfehler modellierter Grössen berücksichtigt und in die Angabe der Aussagesicherheit einbezogen werden.

3. Modelle mit der Struktur von Entscheidungsleitern Alle aufgeführten Beispiele enthalten im Gesamtmodell oder in Teilmodellen als Entscheidungsleitern strukturierte Modelle. Diese Modelltypen spielen in der Angewandten Landschaftsökologie eine grosse Rolle, weil die Vielfalt und Verschiedenartigkeit der zu verknüpfenden Grössen in Gesamtmodellen keine mathematische Formulierung erlaubt (in Teilbausteinen sehr wohl) oder nicht genügend Daten für die Formulierung eines empirisch-statistischen Modells existieren. Abfragemodelle in Form von Entscheidungsleitern sind letztlich immer wissensbasierte Modelle. Hier tut sich noch ein weites Feld zukünftiger landschaftsökologischer Arbeit auf. Solche wissensbasierte Modelle können nämlich vermehrt für Verknüpfungen auf höherer Integrationsebene (siehe Beispiel 4) entwickelt und in eine Anwendungsumgebung integriert werden.

\section{Hohe Integrationsebene}

Landschaftsökologische Aussagen müssen auf einer hohen Integrationsebene liegen. Vielmehr als früher wird diese hohe Integrationsebene heute verfahrensgestützt erreicht. Voraussetzung dafür sind die bessere Datenverfügbarkeit, das bessere Prozessverständnis, die Vielfalt der verfügbaren Einzelmodelle, der Verbund von Kartierung, Modellierung und Messung und die wissensbasierten Modellansätze.

\section{Geographische Informationssysteme als zentrales} Arbeitsinstrument

Alle gezeigten Beispiele beinhalten eine GIS-integrierte oder GIS-gekoppelte Modellierung. Geographische Informationssysteme sind heute ein zentrales Werkzeug in der Landschaftsmodellierung allgemein, der Ableitung landschaftsökologischer Funktionsgrössen, der Bewertung für planerische Fragen und der Visualisierung extern durchgeführter Prozesssimulationen. Die kommerziellen GIS besitzen jedoch entscheidende
Funktionalitäten für eine prozessorientierte Modellierung nicht (z.B.: Fehlen der dritten Dimension und der Zeitdimension). Der Einbezug von Prozessen kann deshalb nur über eine Koppelung von GIS und externen Simulationsmodellen erfolgen. Da sich die Funktionalitäten üblicher GIS-Systeme nur langsam ausweiten werden, müssen sich neue methodische Entwicklungen für landschaftsökologische GIS-Anwendungen sowohl am GIS-internen Einsatz (v.a. in der Praxis üblich) als auch an der unterschiedlich starken Koppelung von GIS und externen Modellen (v.a.in der Forschung üblich) orientieren. Wichtige Felder für neue methodische Entwicklungen sind dabei zum Beispiel geeignete möglichst objektorientierte Datenstrukturen für die Einbindung und Koppelung verschiedener Simulationsmodelle, die Integration wissensbasierter Elemente in Benutzeroberflächen, Modelle zur Ableitung und Visualisierung der Aussagegenauigkeit landschaftshaushaltlicher Raumgrössen, verbesserte Methoden zur Ableitung landschaftsökologischer Steuergrössen (z.B. Ableitung der Bodenbedeckung aus Fernerkundungsdaten) und Konzepte für animierte Karten zur dynamischen Darstellung landschaftshaushaltlicher Prozesse.

\section{Literatur}

ARL (AKADEMIE Für RAUMForschung UND LANDESPLANUNG) (Hrsg.) (1999): Bodenschutz in der Raumordnung. Nachhaltigkeit und Management. = Arbeitsmaterial/Akademie für Raumforschung und Landesplanung 250, Hannover: 1-101.

BACH, M. et al. (1994): Kartierung der potentiellen Filterfunktion von Uferstreifen 2. Teil: Kartierung eines Flusseinzugsgebiets im Mittelgebirgsraum. - In: Z. f. Kulturtechnik u. Landesentwicklung 35: 155-164.

BASTIAN, O. \& M. RöDER (1996): Beurteilung von Landschaftsveränderungen anhand von Landschaftsfunktionen. Untersuchungen am Beispiel zweier Testgebiete im sächsischen Hügelland. - In: Naturschutz und Landschaftsplanung 28 (10): 302-312.

BAuer, A. et al. (1997): Bodenschutz in der Planungspraxis. Dargestellt am Beispiel des Umlandverbandes Frankfurt. - In: Naturschutz und Landschaftsplanung 29 (9): 282-289.

Bronstert, A. et al. (Hrsg.) (1998): Modellierung des Wasser- und Stofftransportes in grossen Einzugsgebieten. PIK Report 43, Potsdam.

Deutscher Rat für LANDespflege (Hrsg.) (1997): Leitbilder für Landschaften in "peripheren Räumen». - = Schriftenreihe des Deutschen Rates für Landespflege 67, Meckenheim: 1-134.

FinKe, L. (1994): Landschaftsökologie. - Das Geographische Seminar, Braunschweig: Westermann: 1-232.

Grunwald, S. \& H.-G. Frede (1999): Using the modified agricultural non-point source pollution model in German watersheds. - In: Catena 37: 319-328. 
Hosmer, D.W. \& S. Lemeshow (1989): Applied Logistic Regression. - New York: John Wiley: 1-307.

LESER, H. (1997): Landschaftsökologie. - Uni-Taschenbücher 521, Stuttgart: Verlag Eugen Ulmer: 1-644.

Mosimann, T. (1999): Angewandte Landschaftsökologie - Inhalte, Stellung und Perspektiven. - In: R. SCHNEIDER-Sliwa et al. (Hrsg.): Angewandte Landschaftsökologie. Grundlagen und Methoden. - Berlin, Heidelberg: Springer: 5-23.

MosimanN, T. (1999): Integration des Bodenschutzes in die Raumplanung. Ziele, Konzepte und Methodik zur Bestimmung besonders schutzwürdiger Bodenflächen am Beispiel des Kantons Basel-Landschaft. - Liestal, Amt für Umweltschutz und Energie: 1-65.

Mosimann, T. et al. (1999): Karten der klima- und immissionsökologischen Funktionen. Instrumente zur prozessorientierten Betrachtung von Klima und Luft in der Umweltplanung. - In: Naturschutz und Landschaftsplanung 31 (4): 101-108.

Mosimann, T. et al. (2001): Entwicklung prozessual begründeter landschaftsökologischer Leitbilder für funktional vielfältige Landschaften. - In: Berichte zur deutschen Landeskunde 75(1), im Druck.

NiEDERSÄCHSISCHES LANDESAMT FÜR ÖKOLOGIE (Hrsg.) (1999): Schutzgut Klima/Luft in der Landschaftsplanung. Bearbeitung der klima- und immissionsökologischen Inhalte im Landschaftsrahmenplan und Landschaftsplan. - Informationsdienst Naturschutz Niedersachsen 19(4), Hildesheim: 201-276.

Prasuhn, V. \& M. Braun (1995): Regional differenzierte Abschwemmung diffuser Phosphor- und Stickstoffeinträge in die Gewässer des Kantons Bern (Schweiz). - In: Z. f. Kulturtechnik und Landesentwicklung 36: 309-314.

Reuter, U. et al. (1991): Luft und Klima als Planungsfaktor im Umweltschutz. Grundlagen für die kommunale Planungs- und Entscheidungspraxis. $-=$ Kontakt und Studium 328, Ehningen b. Böblingen, Expert-Verlag: 1-170.

Schraps, W.G. \& H.P. Schrey (1997): Schutzwürdige Böden in Nordrhein-Westfalen - Bodenkundliche Kriterien für eine flächendeckende Karte zum Bodenschutz. - In: Z. f. Pflanzenernährung, Bodenkunde 106: 407-412.

Sтоск, P. (1992): Synthetische Klimafunktionskarte Ruhrgebiet. - = Kommunalverband Ruhrgebiet (Hrsg.): Arbeitshefte Ruhrgebiet A 040, Essen.

Verband Deutscher Ingenieure VDI (Hrsg.) (1994): Richtlinie $3787 \mathrm{Bl}$. 1: Klima und Lufthygienekarten für Städte und Regionen. - Düsseldorf.

VoGES, J. (1999): Empirisches Modell für die mittlere Massstabsebene zur GIS-gestützten Bestimmung der Anbindung erosionsgefährdeter Ackerflächen an Fliessgewässer. - = Diss. Universität Hannover: 1-227.

WIEGLEB, G. (1997): Leitbildmethode und naturschutzfachliche Bewertung. - In: Z. Ökologie u. Naturschutz 6, Gustav Fischer Verlag: 43-62.
Young, R.A. et al. (1987): AGNPS, Agricultural Nonpoint-source Pollution Model. A watershed analysis tool. - = US Departement of Agriculture/Agricultural Research Service: Conservation Research Report 35: 1-77.

Zimmermann, R. (1988): Zur Ermittlung und Bewertung des Klimas im Rahmen der Landschafts(rahmen)Planung. - = Landesanstalt für Umweltschutz Baden-Württemberg (Hrsg.): Untersuchungen zur Landschaftsplanung 14:1-137.

\section{Zusammenfassung: Angewandte Landschaftsökologie: Der Weg von der Forschung in die Praxis}

Der Unterschied zwischen «Landschaftsökologie» und «Angewandter Landschaftsökologie» ist der Weg zwischen Forschung und Praxis. Angewandte Landschaftsökologie umfasst deshalb mehr als Forschung und Entwicklung. Sie kümmert sich um die Erkenntnisse, Modelle, Verfahren, Konzepte usw. bis sie in der Praxis Eingang gefunden haben. Die entsprechenden Schritte sind in Abb. 1 zusammengefasst. Vier Beispiele aus den Bereichen Landschaftshaushalt, Ressourcenschutz, Raumplanung und Landschaftsentwicklung stellen aktuelle Inhalte, methodische Konzepte, Vorgehensweisen, Modelle und typische Ergebnisse landschaftsökologischer Arbeit dar. Die einzelnen Beispiele werden methodisch eingeordnet, inhaltlich kurz erläutert und mit Karten illustriert. Ein Blick in die Zukunft fasst die grundlegenden Elemente angewandter landschaftsökologischer Arbeit zusammen: Funktionen und Prozesse, Wahrscheinlichkeitsaussagen, wissensbasierte Modelle auf hoher Integrationsebene und GIS als Arbeitsinstrument.

\section{Summary: Applied Landscape Ecology: From research to practical application}

The difference between «landscape ecology» and "applied landscape ecology» is the path taken between research work and practical application. Therefore applied landscape ecology includes more than research and development. It works with scientific findings, models, methods, concepts etc. until they are ready for practical application. The corresponding steps are basic research, calibration and validation, model design, conceptualisation, decision making, development of models for practical application, pilot application and routine application. Four examples from the fields of landscape budget, conservation of natural resources, land use planning and landscape development present current concerns, methodical concepts, procedures, models and typical results of applied landscape ecological research. The various examples are classified methodically, their content is briefly commented and illustrated with maps. A look into the future summarises the basic elements of applied landscape ecological research: functions and processes, pro- 
blems of probability and heterogeneity, knowledge based modelling at a high integration level and GIS as a working instrument.

\section{Résumé: Ecologie du paysage appliquée: la voie de la recherche à la pratique}

La différence entre l'écologie du paysage et l'écologie du paysage appliquée est la voie entre la recherche et la pratique. L'écologie du paysage appliquée englobe donc davantage que la recherche et le développement. Elle s'occupe des résultats, des modèles, des méthodes, des concepts, etc. jusqu'à ce qu'on puisse les utiliser dans la pratique. Les étapes correspondantes sont les suivantes (figure 1): la recherche de base, le calibrage et la validation, l'élaboration de modèles, les concepts, l'aide à la décision, les modèles pour la pratique, l'application pilote et l'application de routine. Quatre exemples tirés des domaines bilan du paysage, protection des ressources, aménagement du territoire et développement du paysage présentent les contenus actuels, les concepts, les méthodes, les modèles et les résultats typiques de l'écologie du paysage. Les divers exemples sont classés méthodiquement, leur contenu est brièvement expliqué et illustré à l'aide de cartes. Un aperçu du futur résume les éléments de base de l'écologie du paysage appliquée: les fonctions et les processus, les problèmes de probabilité et d'hétérogénéité, les modèles basés sur les connaissances (niveau d'intégration élevé) et les SIG comme instrument de travail.

Prof. Dr. Thomas Mosimann, Geographisches Institut der Universität Hannover, Schneiderberg 50, D-30167 Hannover.

e-mail: mosimann@geog.uni-hannover.de 